\title{
A New Two-Lane Lattice Hydrodynamic Model considering the Traffic Interruption Probability under Honk Environment
}

\author{
Qingying Wang $\mathbb{D})^{1,2,3}$ Hongxia Ge $\mathbb{D}^{1,2,3}$ and Rongjun Cheng $\mathbb{D}^{1,2,3}$ \\ ${ }^{1}$ Faculty of Maritime and Transportation, Ningbo University, Ningbo 315211, China \\ ${ }^{2}$ Jiangsu Province Collaborative Innovation Center for Modern Urban Traffic Technologies, Nanjing 210096, China \\ ${ }^{3}$ National Traffic Management Engineering and Technology Research Centre Ningbo University Sub-Centre, \\ Ningbo 315211, China \\ Correspondence should be addressed to Hongxia Ge; gehongxia@nbu.edu.cn
}

Received 26 December 2019; Revised 27 March 2020; Accepted 15 April 2020; Published 13 May 2020

Academic Editor: Chittaranjan Hens

Copyright (c) 2020 Qingying Wang et al. This is an open access article distributed under the Creative Commons Attribution License, which permits unrestricted use, distribution, and reproduction in any medium, provided the original work is properly cited.

\begin{abstract}
By accounting for the traffic interruption probability on two-lane highway under honk environment, an extended lattice hydrodynamic model is presented in the paper. In view of the novel model, a series of researches are carried out. The neutral stability condition and the stable condition can be derived through linear analysis. Then, the mKdV equation near the critical point can be obtained by applying nonlinear analysis, which describes the traffic jams according to the kink-antikink density waves. In addition, numerical simulation is performed, which confirms that traffic interruption probability on two-lane highway under honk environment can develop traffic jams by the change of density waves. Also, the phenomenon is consistent with the results of previous theoretical analysis. It shows that accounting for the traffic interruption probability on two-lane highway under honk environment can stabilize the traffic flow efficiently.
\end{abstract}

\section{Introduction}

In recent years, with the development of social science and technology and the improvement of living standards, the proportion of transportation is getting higher and higher in people's daily life. Therefore, it is not difficult to find that the phenomenon of traffic congestion has become general and severe. From the perspective of theoretical research, the situation as lots of traffic congestion is imbalanced for the traffic system. To explain the evolution traffic congestion formation and improve the stability of traffic flow, scholars at home and abroad have proposed a series of traffic models in recent decades, such as the car-following models $[1,2]$, lattice hydrodynamic models $[3,4]$, and cellular automaton models [5-9].

According to the phenomenon that each vehicle changes the optimal velocity through the following distance of the preceding vehicle, Bando et al. [10] proposed the original car-following model. Furthermore, with the idea of the car- following model, Nagatani [11] put forward the lattice hydrodynamic model for the first time. Different from the carfollowing models, the research direction of lattice hydrodynamic model is the change of traffic flow and density between adjacent interval vehicles. In fact, it is a shift in research direction from micro to macro. On this basis, the study of lattice hydrodynamic model has become more and more colorful, and a lot of factors affecting the traffic flow have been considered, such as driver's anticipation [12-16], driver's visual angle [17], partial reduced lane [18-20], and so on.

In addition, intricate road environment is also one of the most important factors affecting traffic flow. Besides the usual straight roads [21], the current research also includes curved roads [22-25], gradient highway [26], and two-lane highway [27-35]. Among them, the research of two-lane highway is developed recently, which is more coincident with the actual traffic conditions. However, it is relatively few due to the complexity. In 2019, Zhu et al. [27] came up with a 
new lattice hydrodynamic model in two-lane system, considering the empirical lane-changing rate. Qi et al. [28] put forward a lattice hydrodynamic model in 2019, accounting for "backward looking" effect and relative flow information in two-lane highway. Also, Rong and Wen [29] proposed an extended delayed feedback control method for the two-lane traffic flow. And in 2017, Wang et al. [30] proposed a microscopic traffic model, which attempted to reproduce the empirical phenomena of congested traffic flow with an onramp. Following, a coupled map car-following model has been presented, which was extended with feedback control scheme for traffic jam and energy consumption to two-lane traffic system [31]. According to the above analysis, the situation of two-lane highway plays an important role in improving traffic flow. There is no doubt that driver's characteristic effect is also one of the significant factors affecting traffic flow in a complex traffic system, such as honking effect. In the previous research, considering that there is no similar work about it, we propose an improved two-lane lattice hydrodynamic model, which accounts for the traffic interruption probability under honk environment. This paper mainly discusses the impact of lane-changing rate, honking effect, and different driving personalities of drivers on easing traffic congestion, hoping that it can provide some theoretical basis for the implementation of relevant measures in the actual traffic system in the future.

This paper is organized as follows: Section 2 is developed to discuss the new two-lane lattice hydrodynamic model accounting for the traffic interruption probability under honk environment. In Section 3, the stability condition is derived by the use of linear analysis. Following, the $\mathrm{mKdV}$ equation can be obtained by applying nonlinear analysis in Section 4. In Section 5, numerical simulation is performed. Finally, the conclusion is provided in Section 6.

\section{The Modified Lattice Hydrodynamic Model}

In 1999, Nagatani [4] presented a new two-lane lattice hydrodynamic model firstly, which considered lanechanging behaviors compared with the single-lane lattice hydrodynamic model. Figure 1 shows the original two-lane lattice hydrodynamic model of traffic flow, which mainly considers the change of density wave between different lattice caused by vehicle lane change. The above situation can be decreased by the physical models as follows:

$$
\begin{aligned}
& \partial_{t} \rho_{1, j}+\rho_{0}\left(\rho_{1, j} v_{1, j}-\rho_{1, j-1} v_{1, j-1}\right) \\
& =\gamma\left|\rho_{0}^{2} V^{\prime}\left(\rho_{0}\right)\right|\left(\rho_{2, j+1}-2 \rho_{1, j}+\rho_{2, j-1}\right), \\
& \partial_{t} \rho_{2, j}+\rho_{0}\left(\rho_{2, j} v_{2, j}-\rho_{2, j-1} v_{2, j-1}\right) \\
& =\gamma\left|\rho_{0}^{2} V^{\prime}\left(\rho_{0}\right)\right|\left(\rho_{1, j+1}-2 \rho_{2, j}+\rho_{1, j-1}\right),
\end{aligned}
$$

where $\gamma$ is the coefficient constant of lane-changing rate. When the density at site $j$ on lane 1 is lower than the density at site $j-1$ on lane $2, \gamma\left|\rho_{0}^{2} V^{\prime}\left(\rho_{0}\right)\right|\left(\rho_{2, j-1}-\rho_{1, j}\right)$ means the lane-changing rate from lane 2 to lane 1 . From what has been discussed above, when the density at site $j+1$ on lane 2 is lower than that at site $j$ on lane 1 , the lane-changing rate is similar to $\gamma\left|\rho_{0}^{2} V^{\prime}\left(\rho_{0}\right)\right|\left(\rho_{1, j}-\rho_{2, j+1}\right)$ from lane 1 to lane 2 .

Then, we obtain the continuity equation as below by combining equations (1) and (2):

$$
\partial_{t} \rho_{j}+\rho_{0}\left(\rho_{j} v_{j}-\rho_{j-1} v_{j-1}\right)=\gamma\left|\rho_{0}^{2} V^{\prime}\left(\rho_{0}\right)\right|\left(\rho_{j+1}-2 \rho_{j}+\rho_{j-1}\right) \text {, }
$$

where $\rho_{0}$ means the average density of the entire traffic system, $\rho_{j}$ and $v_{j}$ are the local density and velocity at site $j$ on lanes, respectively. In addition, in the two-lane model, we chose $\rho_{j}=\left(\rho_{1, j}+\rho_{2, j}\right) / 2, \rho_{j} v_{j}=\left(\rho_{1, j} v_{1, j}+\rho_{2, j} v_{2, j}\right) / 2$.

So, the evolution equation can be obtained as

$$
\partial_{t} \rho_{j}(t) v_{j}(t)=a \rho_{0} V\left(\rho_{j}(t)\right)-a \rho_{j}(t) v_{j}(t),
$$

where $a$ is the sensitivity of a driver.

The optimal velocity function is

$$
V\left(\rho_{j}(t)\right)=\frac{v_{\max }}{2}\left[\tanh \left(\frac{2}{\rho_{0}}-\frac{\rho_{j}(t)}{\rho_{0}^{2}}-\frac{1}{\rho_{c}}\right)+\tanh \left(\frac{1}{\rho_{c}}\right)\right],
$$

where $v_{\max }$ is the maximal velocity and $\rho_{c}$ means the safety density.

In addition, we also found that the driver would be affected under honk environment. When the current speed of the vehicle is lower than the speed of the vehicle in front, the driver would honk. When the driver hears the horn, he often adjusts his speed according to the traffic conditions he observes. Different characteristics of drivers may have different responses to honking. Considering this, we can assume that the traffic flow would also change. The difference between the maximum traffic and aggressive and timid driving respectively is a probability event, and we assign the probability parameter value $p$ to this event. Because this probability event is random, we call this phenomenon traffic interruption probability in this paper; then the effect can be presented:

$$
\begin{aligned}
\partial_{t} \rho_{j} v_{j}= & a \rho_{0} V\left(\rho_{j+1}\right)-a \rho_{j} v_{j}+a \lambda\left[p\left(Q_{m}-Q_{j}\left(t+\tau_{1}\right)\right)\right. \\
& \left.+(1-p)\left(Q_{m}-Q_{j}\left(t-\tau_{2}\right)\right)\right],
\end{aligned}
$$

where $Q_{m}=\rho_{m} v_{m}$, it means the maximum flux on the traffic system, $\lambda$ is the reaction coefficient, $p$ shows the parameter representing the characteristics strength of drivers in traffic flow, $\tau_{1}$ and $\tau_{2}$ are the reactive time, which correspond to aggressive and timid drivers, respectively. For simplicity, we take $\tau_{1}=\tau_{2}=\lambda \tau$. 


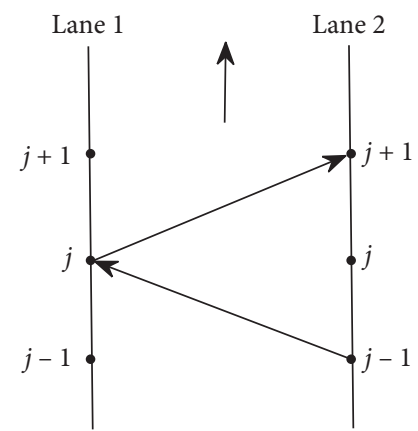

Figure 1: The physical model of traffic flow on two-lane highway.

Eliminating speed $v_{j}$ from (3) and (6), we can obtain the density evolution equation as follows:

$$
\begin{aligned}
{[1} & +a \lambda \tau(2 p-1)] \partial_{t}^{2} \rho_{j}-\gamma\left|\rho_{0}^{2} V^{\prime}\left(\rho_{0}\right)\right|[1+a \lambda \tau(2 p-1)] \partial_{t} \rho_{j+1} \\
& +\left[2 \gamma\left|\rho_{0}^{2} V^{\prime}\left(\rho_{0}\right)\right|(1+a \lambda \tau(2 p-1))+a(\lambda+1)\right] \partial_{t} \rho_{j} \\
& -\gamma\left|\rho_{0}^{2} V^{\prime}\left(\rho_{0}\right)\right|[1+a \lambda \tau(2 p-1)] \partial_{t} \rho_{j-1} \\
& -a(\lambda+1) \gamma\left|\rho_{0}^{2} V^{\prime}\left(\rho_{0}\right)\right|\left(\rho_{j+1}-2 \rho_{j}+\rho_{j-1}\right) \\
& +a \rho_{0}^{2}\left[V\left(\rho_{j+1}\right)-V\left(\rho_{j}\right)\right]=0 .
\end{aligned}
$$

\section{Linear Stability Analysis}

By applying linear analysis, the effect of the traffic interruption probability on two-lane highway under honk environment is investigated. In the steady state of uniform traffic flow, the density and the optimal velocity are both constant values, which are chosen as $\rho_{0}$ and $V\left(\rho_{0}\right)$, respectively. The steady-state solution is

$$
\rho_{j}(t)=\rho_{0}, V\left(\rho_{j}(t)\right)=V\left(\rho_{0}\right) .
$$

A small deviation $y_{j}(t)$ is imposed on the steady-state solution, which is expressed as below:

$$
\rho_{j}(t)=\rho_{0}+y_{j}(t) .
$$

Inserting (8) and (9) into (7), the equation can be obtained:

$$
\begin{aligned}
& {[1+a \lambda \tau(2 p-1)] \partial_{t}^{2} y_{j}(t)} \\
& \quad-\gamma\left|\rho_{0}^{2} V^{\prime}\left(\rho_{0}\right)\right|[1+a \lambda \tau(2 p-1)] \partial_{t} y_{j+1}(t) \\
& \quad+\left[2 \gamma\left|\rho_{0}^{2} V^{\prime}\left(\rho_{0}\right)\right|(1+a \lambda \tau(2 p-1))+a(\lambda+1)\right] \partial_{t} y_{j}(t) \\
& \quad-\gamma\left|\rho_{0}^{2} V^{\prime}\left(\rho_{0}\right)\right|[1+a \lambda \tau(2 p-1)] \partial_{t} y_{j-1}(t) \\
& \quad+a \rho_{0}^{2} V^{\prime}\left(\rho_{0}\right)\left[y_{j+1}(t)-y_{j}(t)\right] \\
& \quad-a(\lambda+1) \gamma\left|\rho_{0}^{2} V^{\prime}\left(\rho_{0}\right)\right|\left(y_{j+1}(t)-2 y_{j}(t)+y_{j-1}(t)\right)=0 .
\end{aligned}
$$

By expanding $y_{j}(t)=A \quad \exp (i k j+z t)$ into (10), we can get

$$
\begin{aligned}
& {[1+a \lambda \tau(2 p-1)] z^{2}-\gamma\left|\rho_{0}^{2} V^{\prime}\left(\rho_{0}\right)\right|[1+a \lambda \tau(2 p-1)] z e^{i k}} \\
& \quad+\left[2 \gamma\left|\rho_{0}^{2} V^{\prime}\left(\rho_{0}\right)\right|(1+a \lambda \tau(2 p-1))+a(\lambda+1)\right] z
\end{aligned}
$$$$
-\gamma\left|\rho_{0}^{2} V^{\prime}\left(\rho_{0}\right)\right|[1+a \lambda \tau(2 p-1)] z e^{-i k}
$$

$$
\begin{aligned}
& +a \rho_{0}^{2} V^{\prime}\left(\rho_{0}\right)\left[(i k)+\frac{1}{2}(i k)^{2}\right] \\
& -a(\lambda+1) \gamma\left|\rho_{0}^{2} V^{\prime}\left(\rho_{0}\right)\right|\left(e^{i k}-2+e^{-i k}\right)=0 .
\end{aligned}
$$

Taking $z=z_{1}(i k)+z_{2}(i k)^{2}+\cdots$, the first- and secondorder terms of the coefficients $(i k)$ and $(i k)^{2}$ can be written as

$$
\begin{aligned}
& z_{1}=-\frac{\rho_{0}^{2} V^{\prime}\left(\rho_{0}\right)}{\lambda+1} \\
& z_{2}=-\frac{[1-a \lambda \tau(2 p-1)] \rho_{0}^{4} V^{\prime 2}\left(\rho_{0}\right)}{a(\lambda+1)^{3}}-\frac{\rho_{0}^{2} V^{\prime}\left(\rho_{0}\right)}{2(\lambda+1)}+\gamma\left|\rho_{0}^{2} V^{\prime}\left(\rho_{0}\right)\right| .
\end{aligned}
$$

According to the above results, it can be found that the unstable region increases and the uniform steady state becomes unstable when $z_{2}<0$. On the contrary, when $z_{2}>0$, the stability region is constantly increasing and the traffic flow maintains a stable state. Therefore, the neutral stability condition for traffic flow is given by 


$$
a=-\frac{2 \rho_{0}^{4} V^{\prime 2}\left(\rho_{0}\right)}{2 \lambda \tau(2 p-1) \rho_{0}^{4} V^{\prime 2}\left(\rho_{0}\right)-(\lambda+1)^{2}\left[2 \gamma\left|\rho_{0}^{2} V^{\prime}\left(\rho_{0}\right)\right|(\lambda+1)-\rho_{0}^{2} V^{\prime}\left(\rho_{0}\right)\right]} .
$$

The stability condition of the model can be obtained by adding disturbance:

$$
a>-\frac{2 \rho_{0}^{4} V^{\prime 2}\left(\rho_{0}\right)}{2 \lambda \tau(2 p-1) \rho_{0}^{4} V^{\prime 2}\left(\rho_{0}\right)-(\lambda+1)^{2}\left[2 \gamma\left|\rho_{0}^{2} V^{\prime}\left(\rho_{0}\right)\right|(\lambda+1)-\rho_{0}^{2} V^{\prime}\left(\rho_{0}\right)\right]}
$$

The neutral stability curves are shown for different values of $p, \lambda$, and $\gamma$, respectively. When $\lambda=0$, the novel model degenerates into the classical two-lane lattice hydrodynamic model proposed by Nagatani [4]. From Figure 2(a), with the increasing of $p$ from 0.1 to 0.3 , the apex of curves decrease when $\lambda=0.1$ and $\gamma=0.1$, this means that the stability area of the traffic flow is increasing. That is to say, it is meaningful to account for the effect of the traffic interruption probability on traffic flow. In Figure 2(b), the change of density curves is also obvious with the change value of $\lambda$ when $p=0.2, \gamma=0.1$. When $\lambda=0.1$, the vertex value of the density curve is the maximum, and it reaches the minimum when the value of $\lambda$ increases to 0.3 . This phenomenon shows the stability of traffic flow can be efficiently enhanced by considering driver's characteristics in the two-lane lattice hydrodynamic model. From Figure 2(c), the stability condition is discussed for different value of $\gamma$ when $p=0.2$ and $\lambda=0.1$. When $\gamma=0.1$, the stability region is the smallest. As the value of $\gamma$ increases to 0.3 , the stability region tends to reach the maximum. In the future research, we can also use the control method to discuss [36]. Above all, we can conclude that the extended two-lane lattice hydrodynamic model is valuable, and the consideration of the traffic interruption probability under honk environment can enhance the stability of traffic flow.

\section{Nonlinear Analysis}

To describe the traffic evolution pattern of the novel model, nonlinear analysis is presented near the critical point $\left(\rho_{c}, a_{c}\right)$ with reductive perturbation method in this section. For $0<\varepsilon \ll 1$, the slow variables are $X$ and $Y$ as follows:

$$
X=\varepsilon(j+b t), \quad T=\varepsilon^{3} t,
$$

where $b$ is an indeterminate constant.

Let $\rho_{j}(t)$ satisfy the equation:

$$
\rho_{j}(t)=\rho_{c}+\varepsilon R(X, T) .
$$

Substituting (15) and (16) into (7) and expending each team to the fifth order of $\varepsilon$, we can obtain

$$
\begin{aligned}
& \varepsilon^{2} k_{1} \partial_{X} R+\varepsilon^{3} k_{2} \partial_{X}^{2} R+\varepsilon^{4}\left(\partial_{T} R+k_{3} \partial_{X}^{3} R+k_{4} \partial_{X} R^{3}\right) \\
& +\varepsilon^{5}\left(k_{5} \partial_{X} \partial_{T} R+k_{6} \partial_{X}^{2} R^{3}+k_{7} \partial_{X}^{4} R\right)=0 .
\end{aligned}
$$

The coefficients $k_{i}(i=1,2,3, \ldots, 7)$ are given in Table 1 where $V^{\prime}=\left.(\partial V(\rho) / \partial \rho)\right|_{\rho=\rho_{c}}$ and $V^{\prime \prime \prime}=\left.\left(\partial^{3} V_{0}(\rho) / \partial \rho^{3}\right)\right|_{\rho=\rho_{c}}$.

Near the critical point $\left(\rho_{c}, a_{c}\right)$, we assume the value of $a_{c}$ as

$$
a_{c}=\left(1+\varepsilon^{2}\right) a .
$$

By taking $b=-\left(\rho_{0}^{2} V^{\prime}\left(\rho_{0}\right) /(1+\lambda)\right)$ and eliminating the second- and third-order terms of $\varepsilon$, we obtain

$$
\begin{aligned}
& \varepsilon^{4}\left(-g_{1} \partial_{X}^{3} R+g_{2} \partial_{X} R^{3}+\partial_{T} R\right) \\
& \quad+\varepsilon^{5}\left(g_{4} \partial_{X}^{4} R+g_{5} \partial_{X}^{2} R^{3}+g_{3} \partial_{X}^{2} R\right)=0,
\end{aligned}
$$

where the coefficients $g_{i}(1,2, \ldots, 5)$ are given in Table 2 .

Equation (19) can be transformed into the standard $\mathrm{mKdV}$ equation by using the following transformations:

$$
\begin{aligned}
& T=\frac{1}{g_{1}} T^{\prime}, \\
& R=\sqrt{\frac{g_{1}}{g_{2}}} R^{\prime} .
\end{aligned}
$$

The $\mathrm{mKdV}$ equation can be obtained with the correction term of $O(\varepsilon)$ :

$$
\begin{aligned}
\partial_{T_{1}} R^{\prime} & =\partial_{X}^{3} R^{\prime}-\partial_{X} R^{\prime 3} \\
& +\varepsilon\left[\frac{g_{3}}{g_{1}} \partial_{X}^{2} R^{\prime}+\frac{g_{4}}{g_{1}} \partial_{X}^{4} R^{\prime}+\frac{g_{5}}{g_{2}} \partial_{X}^{2} R^{\prime 3}\right] .
\end{aligned}
$$

And, the $\mathrm{mKdV}$ equation can be rewritten simply as

$$
\partial_{T}, R^{\prime}=\partial_{X}^{3} R^{\prime}-\partial_{X} R^{\prime 3}+\varepsilon M\left[R^{\prime}\right],
$$

where $M\left[R^{\prime}\right]=\left(g_{3} / g_{1}\right) \partial_{X}^{2} R^{\prime}+\left(g_{4} / g_{1}\right) \partial_{X}^{4} R^{\prime}+\left(g_{5} / g_{2}\right) \partial_{X}^{2} R^{\prime 3}$.

After ignoring the $O(\varepsilon)$, a kink solution of the $\mathrm{mKdV}$ equation can be obtained: 


$$
R_{0}^{\prime}\left(X, T^{\prime}\right)=\sqrt{c} \tanh \left(\sqrt{\frac{c}{2}}\left(X-c T^{\prime}\right)\right),
$$

where $c$ is the propagation velocity.

Subsequently, the value of $c$ should be solved. The following condition needs to be satisfied:

$$
\left(R_{0}^{\prime}, M\left[R_{0}^{\prime}\right]\right) \equiv \int_{-\infty}^{+\infty} \mathrm{d} X \prime R_{0}^{\prime} M\left[R_{0}^{\prime}\right]=0 .
$$

When we consider the correction of $O(\varepsilon)$, and assuming the condition as $R^{\prime}\left(X, T^{\prime}\right)=R_{0}^{\prime}\left(X, T^{\prime}\right)+\varepsilon R_{1}^{\prime}\left(X, T^{\prime}\right)$. The value of $c$ is obtained after solving the integral equation of (24) while $M\left[R_{0}^{\prime}\right]=M[R]$ :

$$
c=\frac{5 g_{2} g_{3}}{2 g_{2} g_{4}-3 g_{1} g_{5}} \text {. }
$$

Substituting all the relevant values that have been solved into (16), the general density kink-antikink solution can be obtained:

$$
\begin{aligned}
\rho_{j}(t)= & \rho_{c}+\sqrt{\frac{g_{1} c}{g_{2}}\left(\frac{a_{c}}{a}-1\right)} \times \tanh \sqrt{\frac{c}{2}\left(\frac{a_{c}}{a}-1\right)} \\
& \times\left[j+\left(1-c g_{1}\right)\left(\frac{a_{c}}{a}-1\right) t\right] .
\end{aligned}
$$

In addition, the amplitude $A$ of the density is

$$
A=\sqrt{\frac{g_{1} c}{g_{2}}\left(\frac{a_{c}}{a}-1\right)} .
$$

From nonlinear analysis above, the $\mathrm{mKdV}$ equation and its kink-antikink solution of the model are obtained, which is closely related to different values of $p, \lambda$, and $\gamma$. It shows the practicality of the new model. Therefore, in the process of studying traffic congestion, we can boldly consider traffic interruption probability on two-lane highway under honk environment, and we have reason to believe that it can effectively alleviate traffic jams.

\section{Numerical Simulation}

To further verify the above results of the theoretical analysis, numerical simulation is discussed to investigate the impact of the traffic interruption probability on a two-lane highway under honk environment. The novel lattice hydrodynamic model of equation (7) is discretized through the difference scheme:

$$
\begin{aligned}
\rho_{j}(t+2 \triangle t)= & \gamma\left|\rho_{0}^{2} V^{\prime}\right| \Delta t\left[\rho_{j+1}(t+\Delta t)-\rho_{j+1}(t)+\rho_{j-1}(t+\Delta t)-\rho_{j-1}(t)\right] \\
& -\triangle t\left[2 \gamma\left|\rho_{0}^{2} V^{\prime}\right|+\frac{a(1+\lambda)}{1+a \lambda \tau(2 p-1)}\right]\left[\rho_{j}(t+\Delta t)-\rho_{j}(t)\right] \\
& +\frac{a(1+\lambda) \triangle t^{2}}{1+a \lambda \tau(2 p-1)} \gamma\left|\rho_{0}^{2} V^{\prime}\right|\left(\rho_{j+1}(t)-2 \rho_{j}(t)+\rho_{j-1}(t)\right)-\rho_{j}(t) \\
& -\frac{a \rho_{0}^{2} \triangle t^{2}}{1+a \lambda \tau(2 p-1)}\left[V\left(\rho_{j+1}(t)\right)-V\left(\rho_{j}(t)\right)\right]+2 \rho_{j}(t+\triangle t) .
\end{aligned}
$$

We can get initial conditions:

$$
\rho_{j}(1)=\rho_{j}(0)= \begin{cases}\rho_{0}, & j \neq \frac{N}{2}, \frac{N}{2}+1, \\ \rho_{0}-0.05, & j=\frac{N}{2}, \\ \rho_{0}+0.05, & j=\frac{N}{2},\end{cases}
$$

where the total number of lattice $N=100, t=10^{4} \mathrm{~s}$, $\rho_{0}=\rho_{c}=0.25, \tau=0.1, a=2.1$.

Figure 3 shows the influence on the traffic system caused by changed value of $p$, which is reflected by the amplitude change of the density curve. When $p=0$, it is clear that the amplitude of the density curve is the highest compared to others in Figure 3(a). Subsequently, in Figures 3(b) to 3(d), the amplitude variation trend of density curves is obvious.
The larger value of $p$ is, the more stable and regular the change of density curves become. The phenomenon can be explained by that the traffic interruption probability is helpful to strengthen the stability of traffic flow. That is to say, the traffic interruption probability can be considered to ease traffic congestion on traffic system.

Figure 4 is the two-dimensional density diagram corresponding to Figure 3 at $t=10300 \mathrm{~s}$. The different values of $p$ for Figures $4(\mathrm{a})$ to $4(\mathrm{~d})$ are taken $0,0.1,0.2,0.3$, respectively. With the increasing value of $p$, the density waves gradually become smooth and uniform. It verifies that the traffic interruption probability is effective to be accounted for two-lane highway under honk environment.

Figure 5 shows the evolution of density waves on traffic system when $p=0.2, \gamma=0.1$, which considers the difference of traffic flow for aggressive and negative drivers under honk environment. From Figures 5(a) to 5(c), the changes of density waves are obvious, which include the decrease of the 


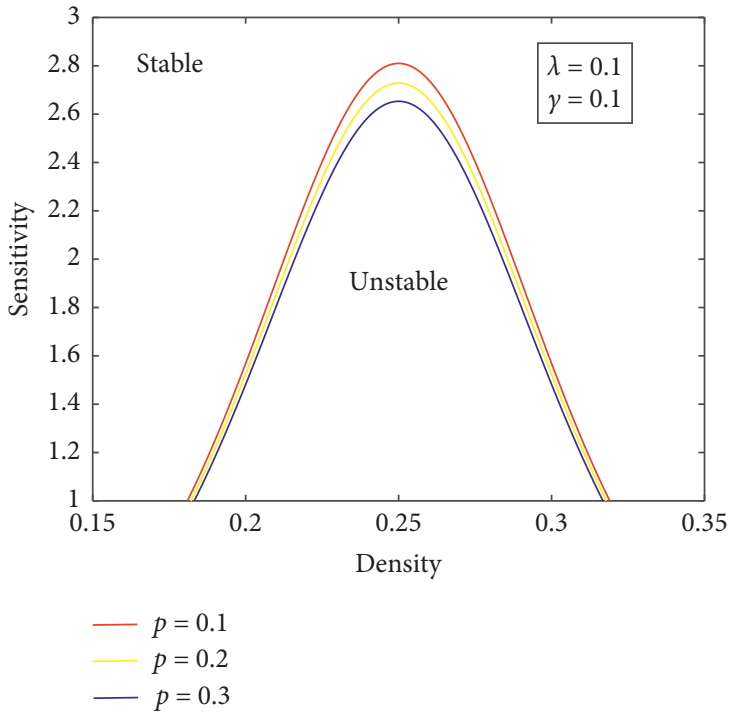

(a)

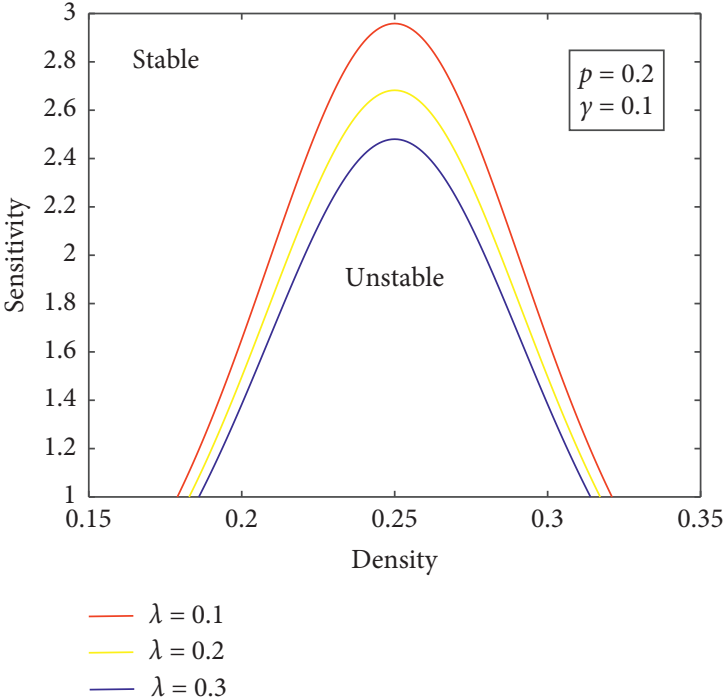

(b)

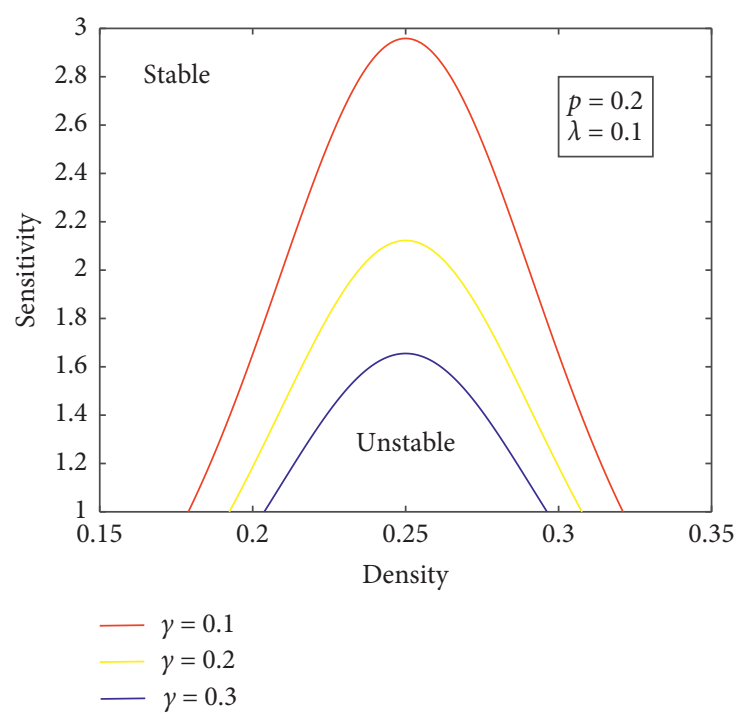

(c)

Figure 2: Phase diagram in $(\rho, a)$ space for (a) $p=0.1,0.2,0.3$ with $\lambda=0.1, \gamma=0.1$. (b) $\lambda=0.1,0.2,0.3$ with $p=0.2$ and $\gamma=0.1$. (c) $\gamma=0.1,0.2,0.3$ with $p=0.2$ and $\lambda=0.1$.

TABLE 1: The coefficients $k_{i}$ of the model.

\begin{tabular}{lc}
\hline Symbol & Corresponding expression \\
\hline$k_{1}$ & $b+\left(\rho_{c}^{2} V^{\prime} /(1+\lambda)\right)$ \\
$k_{2}$ & $\left(\left(2 b^{2}[1+a \lambda \tau(2 p-1)]+a \rho_{c}^{2} V^{\prime \prime \prime}\right) / 2 a(1+\lambda)\right)-\gamma\left|\rho_{c}^{2} V^{\prime}\right|$ \\
$k_{3}$ & $\rho_{c}^{2} V^{\prime} / 6(1+\lambda)$ \\
$k_{4}$ & $\rho_{c}^{2} V^{\prime \prime \prime} / 6(1+\lambda)$ \\
$k_{5}$ & $2 b[1+a \lambda \tau(2 p-1)] / a(1+\lambda)$ \\
$k_{6}$ & $\left(\rho_{c}^{2} V^{\prime}-2(1+\lambda)\left|\rho_{c}^{2} V^{\prime}\right|\right) / 24(1+\lambda)$ \\
$k_{7}$ & $\rho_{c}^{2} V^{\prime \prime \prime} / 12(1+\lambda)$ \\
\hline
\end{tabular}

amplitude and traffic congestion is improved. But it also indicates that the traffic flow has not reached a stable state until the density wave curve becomes a straight line in
TABLE 2: The coefficients $g_{i}$ of the model.

\begin{tabular}{lc}
\hline Symbol & Corresponding expression \\
\hline$g_{1}$ & $-\rho_{c}^{2} V^{\prime} / 6(1+\lambda)$ \\
$g_{2}$ & $\rho_{c}^{2} V^{\prime \prime \prime} / 6(1+\lambda)$ \\
$g_{3}$ & 0 \\
$g_{4}$ & $\left(\rho_{c}^{2} V^{\prime}-2(1+\lambda) \gamma\left|\rho_{c}^{2} V^{\prime}\right|\right) / 24(1+\lambda)$ \\
$g_{5}$ & $\rho_{c}^{2} V^{\prime \prime \prime} / 12(1+\lambda)$ \\
\hline
\end{tabular}

Figure 5(d). At this moment, the value of $\lambda$ satisfies the condition for the extended model to reach a steady state. Therefore, we can conclude that considering aggressive and 


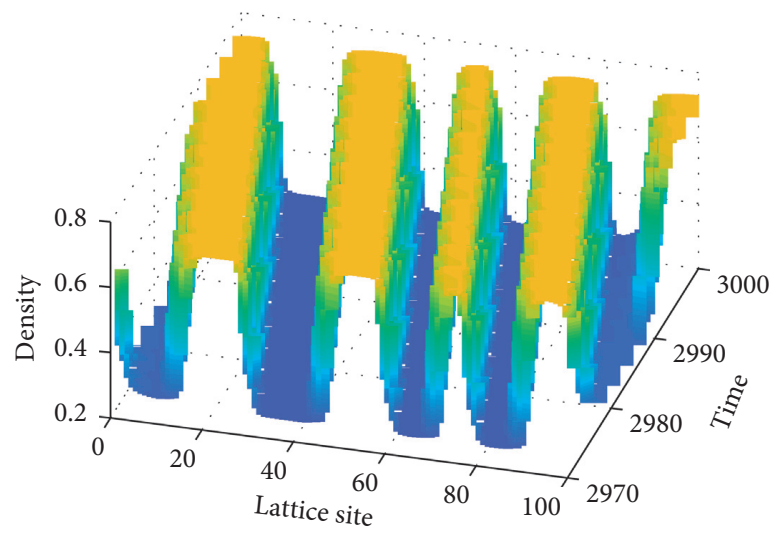

(a)

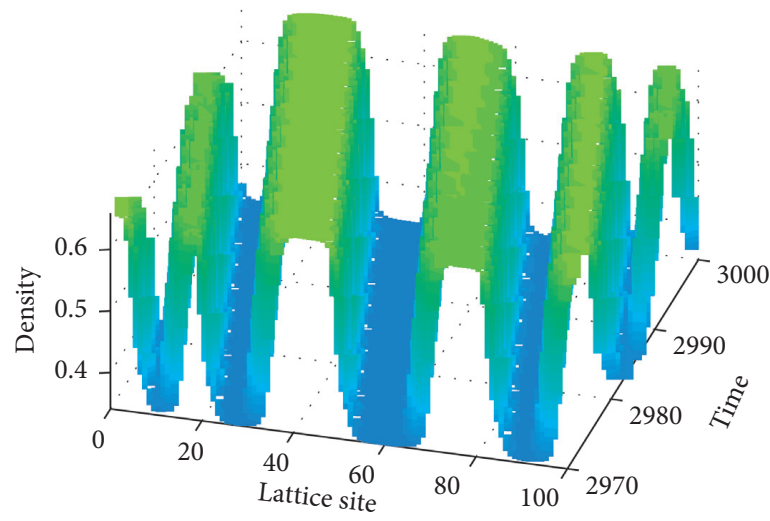

(c)

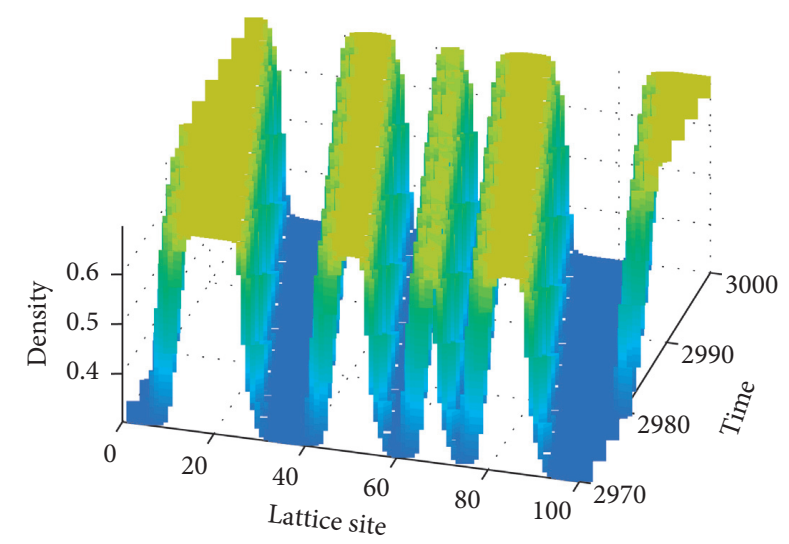

(b)

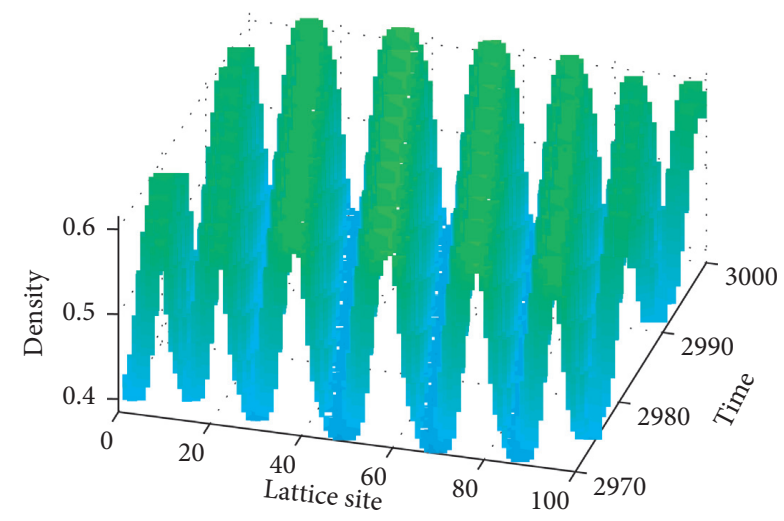

(d)

Figure 3: The phase diagram of the model according to different values of parameter $p$. (a) $p=0$, (b) $p=0.1,(\mathrm{c}) p=0.2$, (d) $p=0.3$.

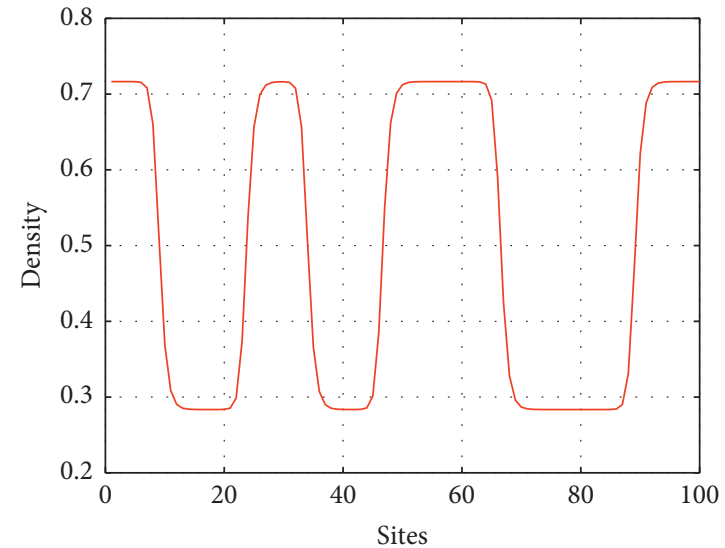

(a)

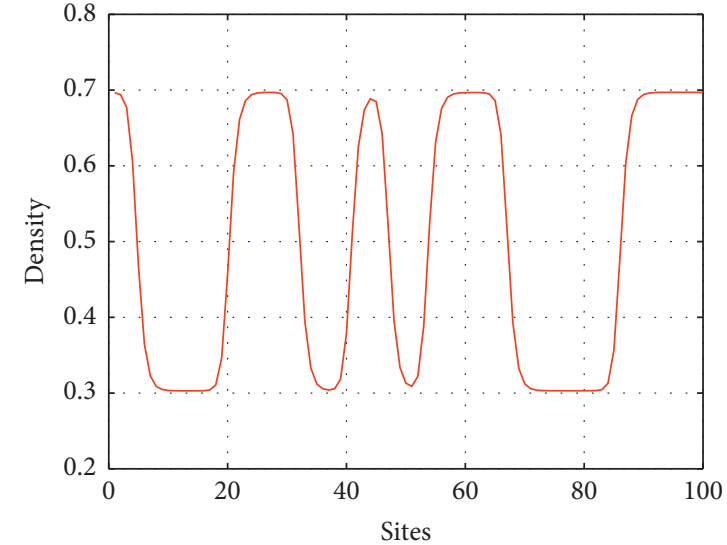

(b)

Figure 4: Continued. 


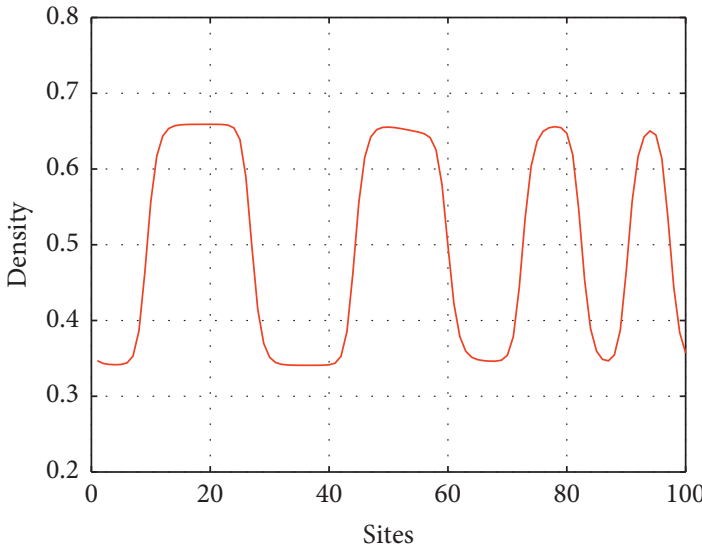

(c)

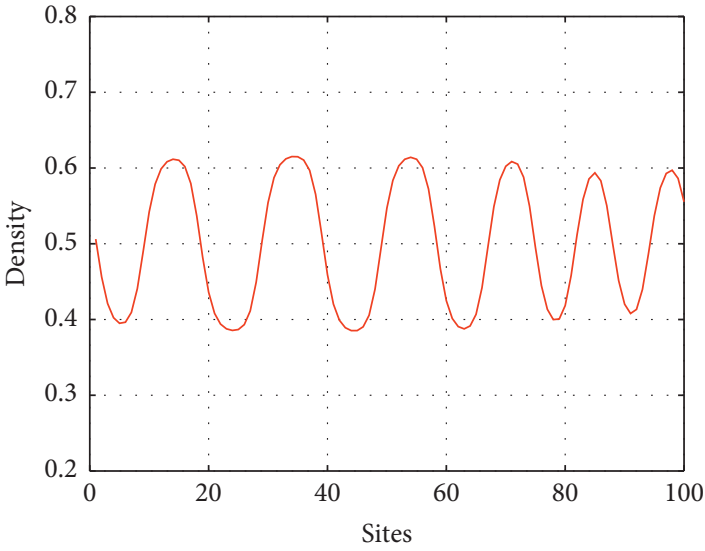

(d)

Figure 4: Density profile of the density wave at $t=10300 \mathrm{~s}$ corresponds to Figure 3. (a) $p=0$, (b) $p=0.1$, (c) $p=0.2$, (d) $p=0.3$.

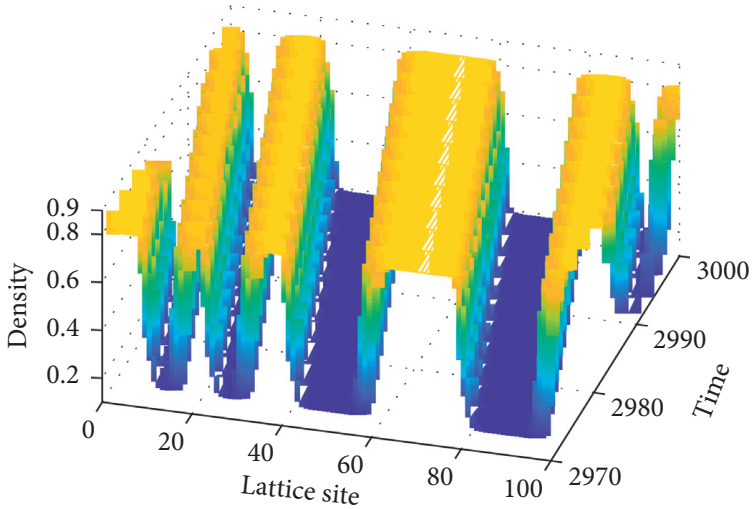

(a)

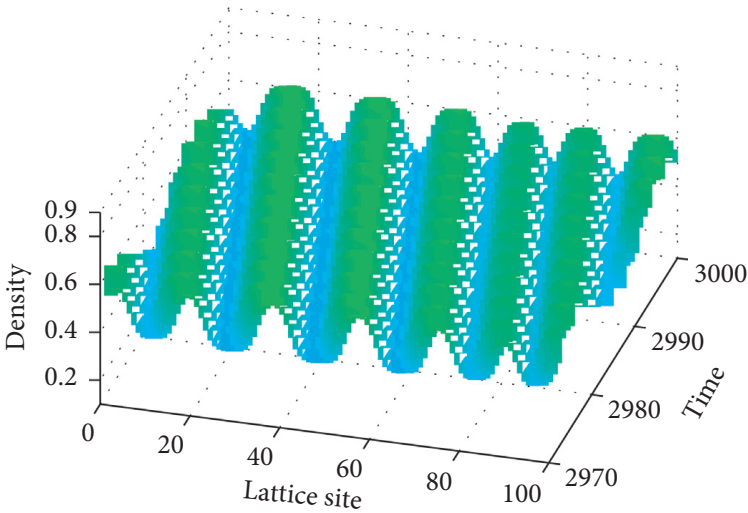

(c)

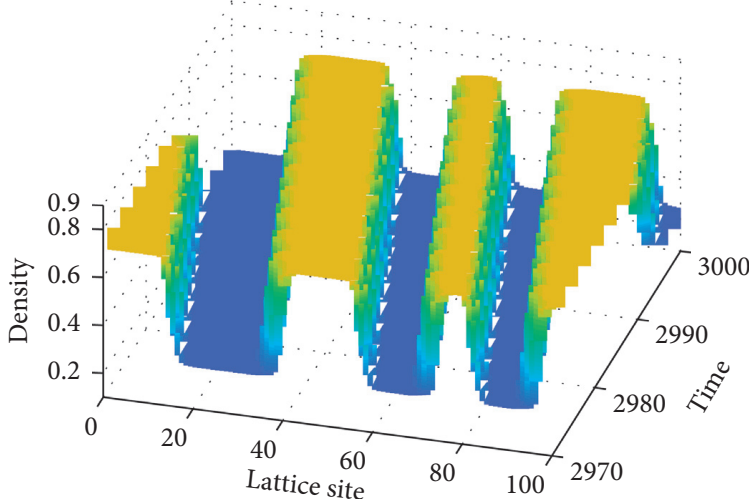

(b)

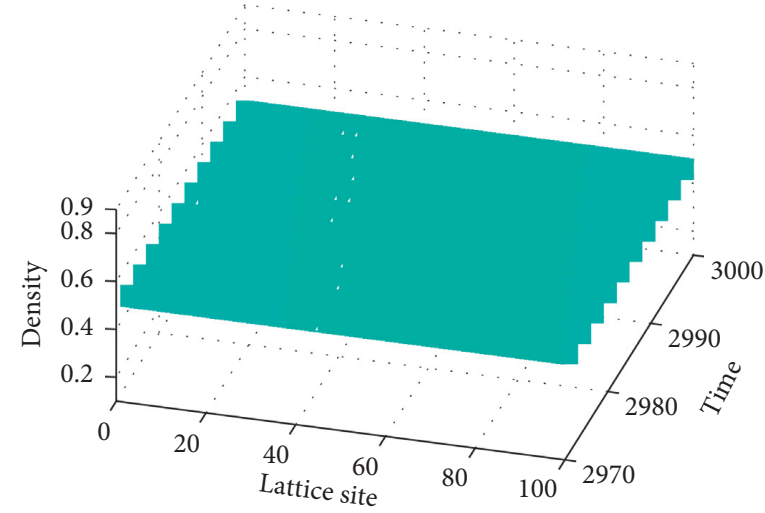

(d)

Figure 5: The phase diagram of the model according to different values of parameter $\lambda$. (a) $\lambda=0$, (b) $\lambda=0.1$, (c) $\lambda=0.2$, (d) $\lambda=0.3$.

negative drivers in two-lane highway under honk environment is necessary.

Figure 6 shows the density profile for fixed time $t=10300 \mathrm{~s}$, which corresponds to Figure 5 . The amplitudes of the density waves decreased until it reached a steady state. It means traffic jams can be improved when accounting for aggressive and negative drivers in two-lane highway under honk environment.

From Figure 7, the traffic flow can be improved gradually from Figures $7(a)$ to $7(d)$, it shows different values of lanechanging rate $\gamma$ under $p=0.2, \lambda=0.1$. According to Figure $7(\mathrm{a})$, the kink-antikink density wave is strong, and the 


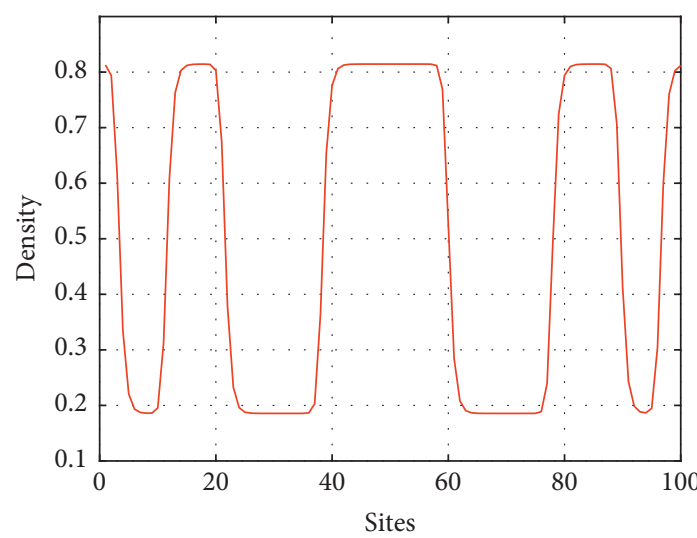

(a)

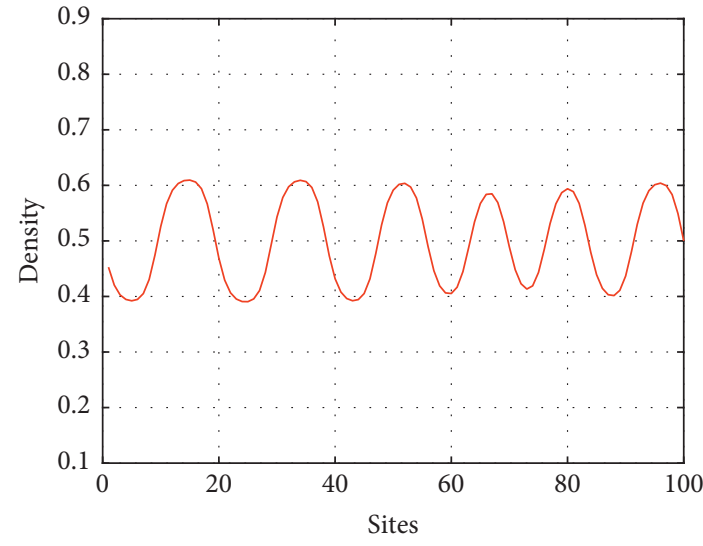

(c)

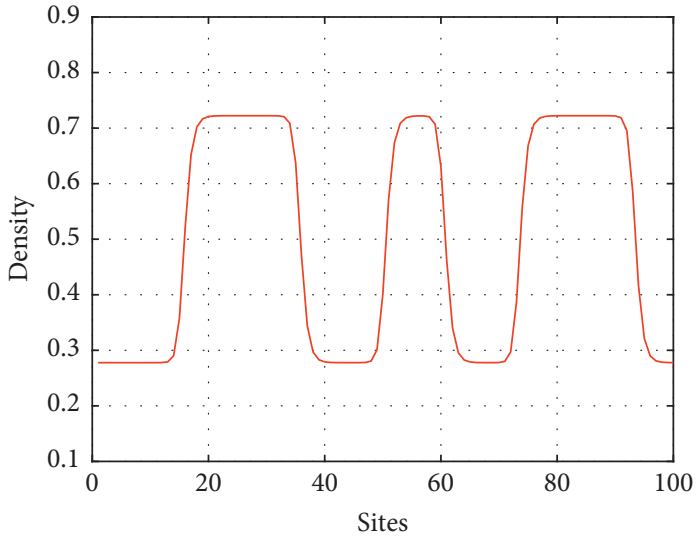

(b)

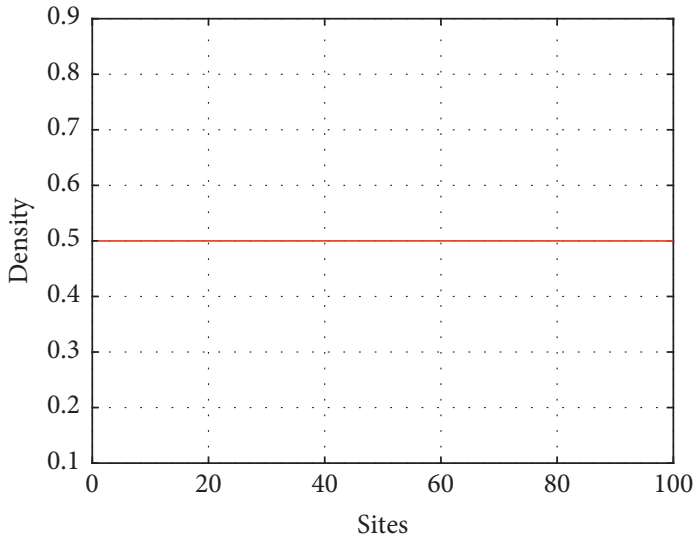

(d)

Figure 6: Density profile of the density wave at $t=10300 \mathrm{~s}$ corresponds to Figure 5 . (a) $\lambda=0$, (b) $\lambda=0.1$, (c) $\lambda=0.2$, (d) $\lambda=0.3$.

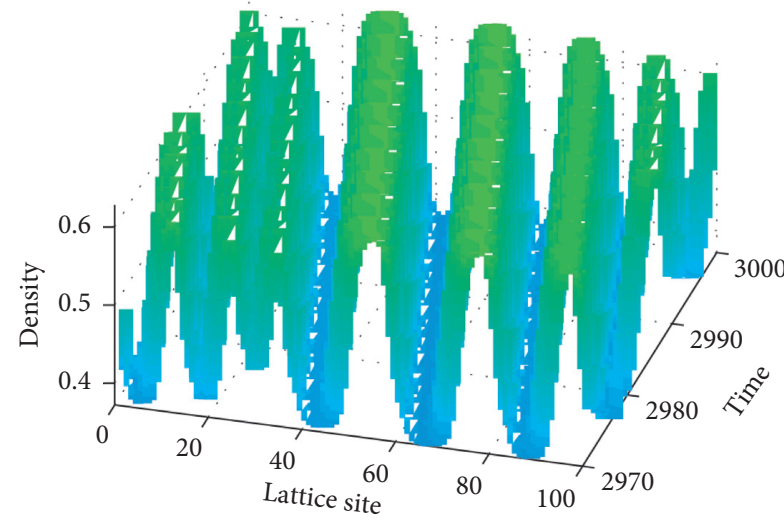

(a)

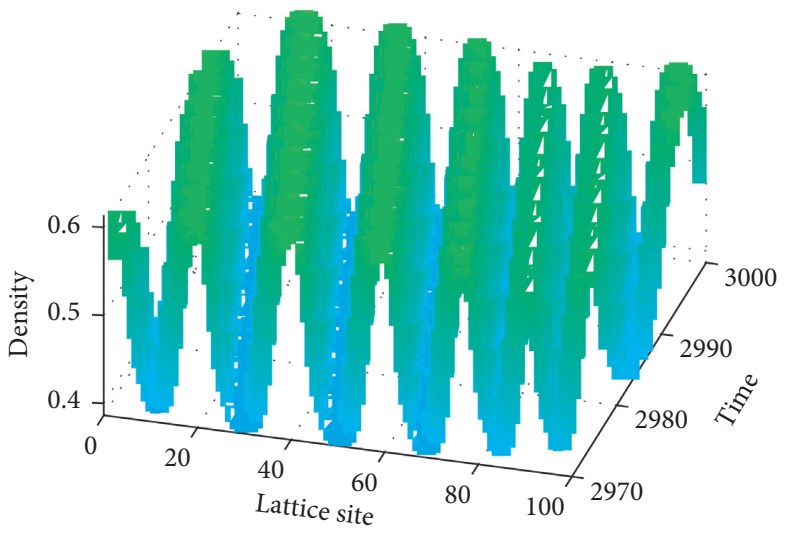

(b)

FIGURE 7: Continued. 


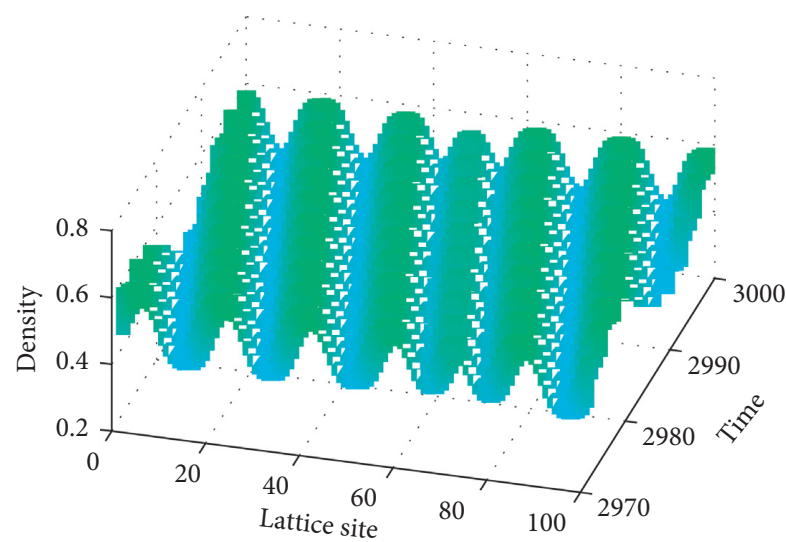

(c)

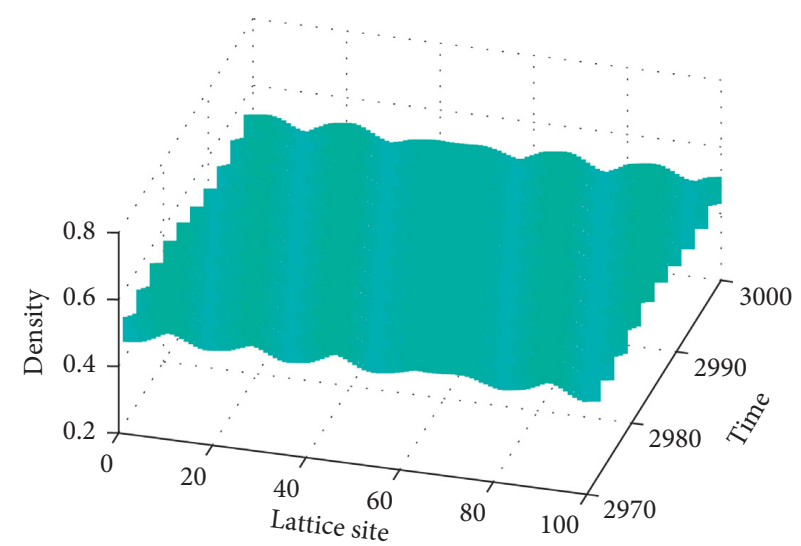

(d)

Figure 7: The phase diagram of the model according to different values of parameter $\gamma$. (a) $\gamma=0$, (b) $\gamma=0.1$, (c) $\gamma=0.2$, (d) $\gamma=0.3$.

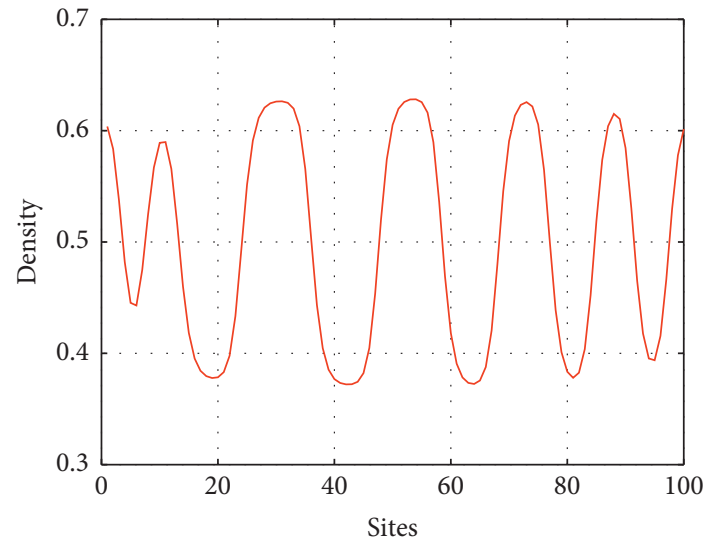

(a)

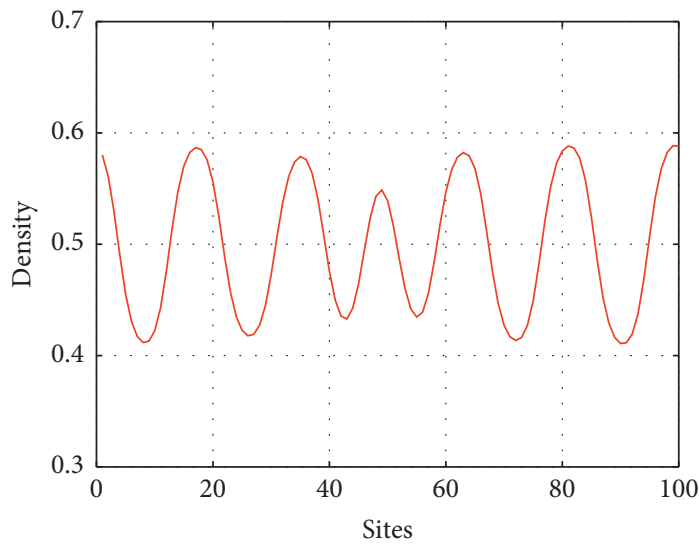

(c)

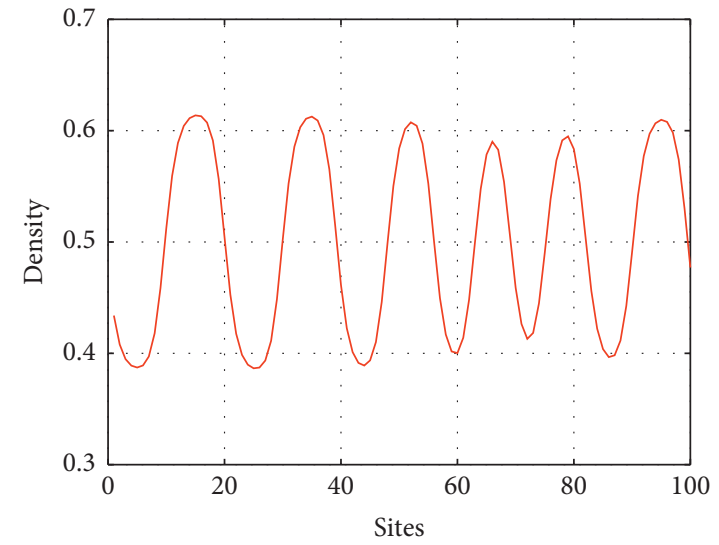

(b)

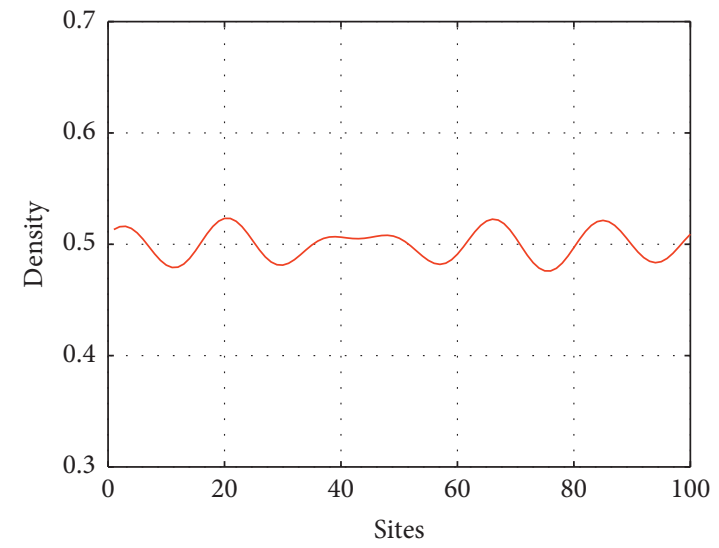

(d)

Figure 8: Density profile of the density wave at $t=10300$ s corresponds to Figure 7. (a) $\gamma=0$, (b) $\gamma=0.1$, (c) $\gamma=0.2$, (d) $\gamma=0.3$.

traffic flow is unstable. When increasing the value of $\gamma$ from 0 to 0.3 , it is found that the amplitudes of density waves reduce gradually. This phenomenon indicates that the steady state of traffic flow is approaching the steady state, and the traffic flow will reach the final stable state at future. Regarding the lane-changing rate, the different study proposed by Wang et al. [37] also reached the same conclusion. That is to say, the consideration of lane-changing in two-lane system is valuable to reduce traffic jams.

Figure 8 shows the density profile at $t=10300 \mathrm{~s}$, which corresponds to Figure 7. It is apparent that the amplitudes of density waves reduce when we increase the number of $\gamma$ in 
two-lane system. It indicates that the effect of two-lane lattice hydrodynamic model can suppress the traffic jams more effectively.

\section{Conclusion}

The paper proposed an extended lattice hydrodynamic model, which considers the traffic interruption probability on two-lane highway under honk environment. To demonstrate the reachability of the model, a series of theoretical analysis and numerical simulations of the extended model are carried out. By linear analysis, the stability condition and stability curves are shown. And the kink-antikink solition solution for the $\mathrm{mKdV}$ equation can be obtained. Then, the evolution is studied in numerical simulation with different values of the traffic interruption probability, the control constant of the honking effect, and lane-changing rate. The variation of density curves show that the new model is researchable, which is consistent with the results of linear analysis and nonlinear analysis. We can conclude that the lattice hydrodynamic model considering the traffic interruption probability on two-lane highway under honk environment is effective, it is beneficial to enhance traffic jams on traffic system.

\section{Data Availability}

The data used to support the findings of this study are available from the corresponding author upon request.

\section{Conflicts of Interest}

The authors declare that they have no conflicts of interest.

\section{Acknowledgments}

This work was supported by the Program of Humanities and Social Science of Education Ministry of China (Grant no. 20YJA630008), the Natural Science Foundation of Zhejiang Province, China (Grant no. LY20G010004), the National Key Research and Development Program of China-Traffic Modeling, Surveillance and Control with Connected \&Automated Vehicles (Grant no. 2017YFE9134700), and the K.C. Wong Magna Fund in Ningbo University, China.

\section{References}

[1] X. Wang, R. Jiang, L. Li, Y.-L. Lin, and F.-Y. Wang, "Long memory is important: a test study on deep-learning based carfollowing model," Physica A: Statistical Mechanics and Its Applications, vol. 514, pp. 786-795, 2019.

[2] Y. Zhang, W. Xiang, J. Dong, and W. Wang, "An improved car-following model considering forecast speed difference with delay time," Modern Physics Letters B, vol. 33, no. 33, Article ID 1950414, 2019.

[3] Q. Wang and H. Ge, "An improved lattice hydrodynamic model accounting for the effect of "backward looking" and flow integral," Physica A: Statistical Mechanics and Its Applications, vol. 513, pp. 438-446, 2019.

[4] T. Nagatani, "Jamming transitions and the modified Korteweg-de Vries equation in a two-lane traffic flow," Physica A:
Statistical Mechanics and Its Applications, vol. 265, no. 1-2, pp. 297-310, 1999.

[5] S. C. Bruningk, P. Ziegenhein, I. Rivens, U. Oelfke, and G. ter Haar, "Cellular automaton model for spheroid response to radiation and hyperthermia treatments," Scientific Reports, vol. 9, no. 1, p. 17674, 2019.

[6] Z. Wang, S. Ma, R. Jiang, and J. Tian, "A cellular automaton model reproducing realistic propagation speed of downstream front of the moving synchronized pattern," Transportmetrica B: Transport Dynamics, vol. 7, no. 1, pp. 295-310, 2019.

[7] V. Sharma, L. Braud, and M. Lehning, "Understanding snow bedform formation by adding sintering to a cellular automata model," The Cryosphere, vol. 13, no. 12, pp. 3239-3260, 2019.

[8] Y. Chen, X. Li, X. Liu, H. Huang, and S. Ma, "Simulating urban growth boundaries using a patch-based cellular automaton with economic and ecological constraints," International Journal of Geographical Information Science, vol. 33, no. 1, pp. 55-80, 2019.

[9] L. Wei, Y. Cao, X. Lin, M. Wang, and W. Huang, "Quantitative cellular automaton model and simulations of dendritic and anomalous eutectic growth," Computational Materials Science, vol. 156, pp. 157-166, 2019.

[10] M. Bando, K. Hasebe, A. Nakayama, A. Shibata, and Y. Sugiyama, "Dynamical model of traffic congestion and numerical simulation," Physical Review E, vol. 51, no. 2, pp. 1035-1042, 1995.

[11] T. Nagatani, "Modified KDV equation for jamming transition in the continuum models of traffic," Physica A: Statistical Mechanics and Its Applications, vol. 261, no. 3-4, pp. 599-607, 1998.

[12] A. K. Gupta and P. Redhu, “Analyses of the driver's anticipation effect in a new lattice hydrodynamic traffic flow model with passing," Nonlinear Dynamics, vol. 76, no. 2, pp. 1001-1011, 2014.

[13] D.-H. Sun, G. Zhang, M. Zhao, S.-L. Cheng, and J.-D. Cao, "Stability analysis of feedforward anticipation optimal flux difference in traffic lattice hydrodynamic theory," Communications in Nonlinear Science and Numerical Simulation, vol. 56, pp. 287-295, 2018.

[14] E. Muhrer and M. Vollrath, "The effect of visual and cognitive distraction on driver's anticipation in a simulated car following scenario," Transportation Research Part F: Traffic Psychology and Behaviour, vol. 14, no. 6, pp. 555-566, 2011.

[15] S. Sharma, "Effect of driver's anticipation in a new two-lane lattice model with the consideration of optimal current difference," Nonlinear Dynamics, vol. 81, no. 1-2, pp. 991-1003, 2015.

[16] R. J. Cheng, H. X. Ge, and J. F. Wang, “The nonlinear analysis for a new continuum model considering anticipation and traffic jerk effect," Applied Mathematics and Computation, vol. 332, pp. 493-505, 2018.

[17] L. Zheng and Z. He, "A new car following model from the perspective of visual imaging," International Journal of Modern Physics C, vol. 26, no. 8, p. 1550090, 2015.

[18] A.-K. Kraft, F. Naujoks, J. Wörle, and A. Neukum, "The impact of an in-vehicle display on glance distribution in partially automated driving in an on-road experiment," Transportation Research Part F: Traffic Psychology and Behaviour, vol. 52, pp. 40-50, 2018.

[19] C. T. Jiang, R. J. Cheng, and H. X. Ge, "Mean-field flow difference model with consideration of on-ramp and offramp," Physica A: Statistical Mechanics and its Applications, vol. 513, pp. 465-476, 2019. 
[20] T. Li, F. Hui, and X. Zhao, "An improved car-following model considering the impact of safety messages," Modern Physics Letters B, vol. 32, no. 32, Article ID 1850398, 2018.

[21] W.-X. Zhu, D. Jun, and L.-D. Zhang, "A compound compensation method for car-following model," Communications in Nonlinear Science and Numerical Simulation, vol. 39, pp. 427-441, 2016.

[22] R. Cheng and Y. Wang, "An extended lattice hydrodynamic model considering the delayed feedback control on a curved road," Physica A: Statistical Mechanics and Its Applications, vol. 513, pp. 510-517, 2019.

[23] J.-L. Cao and Z.-K. Shi, "A novel lattice traffic flow model on a curved road," International Journal of Modern Physics C, vol. 26, no. 11, Article ID 1550121, 2015.

[24] J. Zhou and Z.-K. Shi, "Lattice hydrodynamic model for traffic flow on curved road," Nonlinear Dynamics, vol. 83, no. 3, pp. 1217-1236, 2016.

[25] Y.-D. Jin, J. Zhou, Z.-K. Shi, H.-L. Zhang, and C.-P. Wang, "Lattice hydrodynamic model for traffic flow on curved road with passing," Nonlinear Dynamics, vol. 89, no. 1, pp. 107-124, 2017.

[26] D.-H. Sun, P. Tan, D. Chen, F. Xie, and L.-H. Guan, "Burgers and $\mathrm{mKdV}$ equation for car-following model considering drivers' characteristics on a gradient highway," Modern Physics Letters B, vol. 32, no. 26, Article ID 1850314, 2018.

[27] C. Zhu, S. Zhong, and S. Ma, "Two-lane lattice hydrodynamic model considering the empirical lane-changing rate," Communications in Nonlinear Science and Numerical Simulation, vol. 73, pp. 229-243, 2019.

[28] X. Qi, R. Cheng, and H. Ge, "Nonlinear analysis of a new twolane lattice hydrodynamic model accounting for "backward looking" effect and relative flow information," Modern Physics Letters B, vol. 33, no. 19, Article ID 1950223, 2019.

[29] Y. Rong and H. Y. Wen, "An extended delayed feedback control method for the two-lane traffic flow," Nonlinear Dynamics, vol. 94, no. 4, pp. 2479-2490, 2019.

[30] T. Wang, J. Zhang, Z. Gao, W. Zhang, and S. Li, "Congested traffic patterns of two-lane lattice hydrodynamic model with on-ramp," Nonlinear Dynamics, vol. 88, no. 2, pp. 1345-1359, 2017.

[31] H.-D. He, C.-Y. Zhang, W.-L. Wang, Y.-Y. Hao, and Y. Ding, "Feedback control scheme for traffic jam and energy consumption based on two-lane traffic flow model," Transportation Research Part D: Transport and Environment, vol. 60, pp. 76-84, 2018.

[32] S. Sharma, "Lattice hydrodynamic modeling of two-lane traffic flow with timid and aggressive driving behavior," Physica A: Statistical Mechanics and Its Applications, vol. 421, pp. 401-411, 2015.

[33] N. Madaan and S. Sharma, "Effects of multi-phase optimal velocity function on a lattice model accounting for driver's behavior," Nonlinear Dynamics, vol. 80, pp. 1091-1108, 2015.

[34] D. Kaur and S. Sharma, "The impact of the predictive effect on traffic dynamics in a lattice model with passing," European Physical Journal B, vol. 93, p. 35, 2020.

[35] D. Kaur and S. Sharma, "A new two-lane lattice model by considering predictive effect in traffic flow," Physica A: Statistical Mechanics and Its Applications, vol. 539, Article ID 122913, 2020.

[36] Y. Sun, H. Ge, and R. Cheng, "An extended car-following model considering driver's memory and average speed of preceding vehicles with control strategy," Physica A: Statistical Mechanics and Its Applications, vol. 521, pp. 752-761, 2019.
[37] T. Wang, R. Cheng, and H. Ge, "Analysis of a novel two-lane lattice hydrodynamic model considering the empirical lane changing rate and the self-stabilization effect," IEEE Access, vol. 7, pp. 174725-174733, 2019. 